\title{
Exposure assessment of phthalate esters in Japanese pregnant women by using urinary metabolite analysis
}

\author{
Yayoi Suzuki $\cdot$ Mayu Niwa $\cdot$ Jun Yoshinaga $\cdot$ \\ Chiho Watanabe - Yoshifumi Mizumoto • \\ Shigeko Serizawa $\cdot$ Hiroaki Shiraishi
}

Received: 25 September 2008/ Accepted: 12 January 2009/Published online: 18 February 2009

(C) The Japanese Society for Hygiene 2009

\begin{abstract}
Objectives Our objectives were (1) to evaluate whether single spot urine is suitable media for longer-term phthalate esters exposure assessment, and (2) to estimate intake level of phthalate esters of Japanese pregnant women using urinary metabolites as an indicator of prenatal exposure level in their offspring.

Methods We analyzed nine metabolites (MMP, MEP, MnBP, MBzP, MEHP, MEOHP, MEHHP, MINP, MnOP) of seven phthalate esters in spot urine samples from 50 pregnant women by high-performance liquid chromatography-tandem mass spectrometry (HPLC-MS/MS). Using four urine samples collected from each of 12 subjects from 50 pregnant women within 5-12 weeks, we compared intra- and interindividual variation in urinary metabolites by calculation of intraclass correlation coefficient (ICC). We estimated daily intakes of 50 pregnant women from their urinary metabolite concentrations.
\end{abstract}

Y. Suzuki $(\bowtie) \cdot$ M. Niwa $\cdot$ J. Yoshinaga

Department of Environmental Studies,

The University of Tokyo, Kashiwanoha

5-1-5, Kashiwa, Chiba 270-8563, Japan

e-mail: y_suzuki@envhlth.k.u-tokyo.ac.jp

C. Watanabe

Department of Human Ecology,

The University of Tokyo, Tokyo, Japan

Y. Mizumoto

Department of Obstetrics and Gynecology,

Central Hospital of Self-Defense Force, Tokyo, Japan

S. Serizawa $\cdot$ H. Shiraishi

National Institute for Environmental Studies, Ibaraki, Japan
Results ICCs for seven phthalate metabolite concentrations in single spot urine samples were: MMP (0.57), MEP (0.47), MnBP (0.69), MBzP (0.28), MEHP (0.51), MEHHP (0.43), and MEOHP (0.41) in 12 pregnant women. Phthalate ester metabolites had high detection rates in 50 subjects. The mean daily intake ranged from 0.01 to $2 \mu \mathrm{g} / \mathrm{kg}$ per day. The daily intake levels in all subjects were lower than corresponding tolerable daily intake (TDI) set by the European Food Safety Authority (EFSA), though maximum value for DnBP of $6.91 \mu \mathrm{g} / \mathrm{kg}$ per day accounted for $70 \%$ of TDI value.

Conclusions Higher ICCs indicated that phthalate metabolite levels in single spot urine could reflect longer-term exposure to the corresponding diesters of subjects. Although the current exposure level was less than TDIs, further studies and exposure monitoring are needed to reveal the toxicity of phthalate esters to sensitive subpopulation.

Keywords Phthalate esters · Biomarkers - Urinary metabolites - Intraclass correlation coefficient .

Daily intake

$\begin{array}{ll}\text { Abbreviations } \\ \text { MMP } & \text { Monomethyl phthalate } \\ \text { MEP } & \text { Monoethyl phthalate } \\ \text { MnBP } & \text { Mono- } n \text {-butyl phthalate } \\ \text { MBzP } & \text { Monobenzyl phthalate } \\ \text { MEHP } & \text { Mono-2-ethylhexyl phthalate } \\ \text { MEHHP } & \text { Mono-(2-ethyl-5-hydroxyhexyl)phthalate } \\ \text { MEOHP } & \text { Mono-(2-ethyl-5-oxohexyl)phthalate } \\ \text { MINP } & \text { Mono-iso-nonyl phthalate } \\ \text { MnOP } & \text { Mono- } n \text {-octyl phthalate } \\ \text { MOE } & \text { The Ministry of the Environment in Japan } \\ \text { EFSA } & \text { European Food Safety Authority }\end{array}$




\section{Introduction}

Phthalate esters are produced in abundance all over the world because of their multiple uses. For instance, di(2ethylhexyl)phthalate (DEHP) has been produced as one of the major plasticizers for many years [1]. Other phthalate esters, such as di-n-butyl phthalate (DnBP) and diethyl phthalate (DEP), are used in paints and consumer products, and as plasticizers $[2,3]$. Taking the abundance of plastics and other phthalate-ester-containing materials in our immediate environment into consideration, it is highly probable that humans are exposed to various phthalate esters in daily life.

The recognition that some phthalate esters have adverse effects on male reproductive system in experimental animals, especially via in utero exposure, has been increasing [4]. Testosterone production of fetal male rodent is reduced following in utero phthalate ester exposure, which results in malformation of male reproductive tract, such as reduced anogenital distance (AGD), retained nipples, hypospadias, cleft phallus, undescended testis epididymal agenesis, and testicular atrophy [5, 6].

Moreover, several epidemiological studies have suggested adverse health effects that were similar to reproductive toxicities observed in experimental animals [7-9]; for example, Swan et al. reported the relationship between mother's phthalate ester exposure levels in gestational period and their male newborn's anogenital distance index (AGI). This report suggested that prenatal phthalate ester exposure could affect human reproductive health. They also speculated that humans might be more sensitive than experimental animals to reproductive effect of phthalate ester because the exposure levels of the human subjects in that report were very low compared with those administered to animals in the in vivo experiments [8]. Therefore, it has become much more important to evaluate phthalate ester exposure levels in human population of sensitive period (prenatal, newborn, and infant), and also to assess human health effects caused by exposure.

Once phthalate esters are taken into human body, they are rapidly metabolized to monoesters by hydrolysis and oxidation and then are conjugated with glucuronide; subsequently most of the phthalate esters are excreted in the urine within $24 \mathrm{~h}[10,11]$. This metabolic process can to a certain extent differ due to the length of alkyl chain of the phthalate esters; for example, phthalate esters with shorter alkyl chain are mainly excreted as monoesters, while those with longer alkyl chain are further metabolized to more hydrophilic oxidative form [11].

Since Blount et al. first reported a sensitive analytical method for phthalate ester metabolites in human urine using high-performance liquid chromatography-tandem mass spectrometry (HPLC-MS/MS) [12], exposure assessment of phthalate esters has become routine. Although single spot urine sample was usually used for exposure assessment [7, 13-17], concern has been raised over whether monoester concentration in single spot urine represents the subject's long-term exposure to phthalate esters because biological half-life in human body is short. Hoppin et al. [18], Hauser et al. [19], and Teitelbaum et al. [20] reported utility of single spot urine for phthalate esters exposure assessment; however, Fromme et al. [21] did not. Hence it is still unclear whether spot urine is usable for human exposure assessment of phthalate esters.

The main purposes of the present study are (1) to evaluate whether single spot urine is suitable media for the assessment of longer-term phthalate ester exposure, and (2) to estimate intake level of phthalate esters of Japanese pregnant women using urinary metabolite concentrations as an indicator of prenatal exposure level of their offspring.

\section{Materials and methods}

\section{Participants}

Fifty healthy Japanese pregnant women, who were outpatients of a department of obstetrics and gynecology of a hospital in Tokyo, participated in this study during 20052006. They were randomly chosen from outpatients who had no pathological symptoms as diagnosed by a gynecologist. The subjects agreed to participate after being explained the purpose and the procedure of this study by the gynecologist. Ethical Committee of the hospital approved this study.

\section{Sampling}

We collected single spot urine samples from the subjects at one of their regular maternal health check-ups. Sampling was done at 25-40 gestational weeks. The urine samples were collected in polypropylene (PP) bottle that was washed with ultrapure water and methanol prior to use, and stored at $-20^{\circ} \mathrm{C}$ until analysis. One spot urine sample was collected from all of the 50 subjects to assess phthalate exposure during pregnancy. Additional three urine samples were collected with 1-6 weeks interval from 12 of the 50 subjects to evaluate intra- and interindividual variation of urinary phthalate metabolite concentrations.

\section{Chemicals}

Standard solutions $(100 \mu \mathrm{g} / \mathrm{ml})$ of nine phthalate monoesters (monomethyl, monoethyl, mono-n-butyl, monobenzyl, 
monoisononyl, mono- $n$-octyl, mono-2-ethylhexyl, mono-2ethyl-5-hydroxyhexyl, mono-2-ethyl-5-oxohexyl) (>98\% purity) were purchased from Cambridge Isotope Laboratories, Inc. (Andover, MA). ${ }^{13} \mathrm{C}_{4}$-labeled phthalate monoesters were purchased from Cambridge Isotope Laboratories, Inc. (Andover, MA) for an internal standard. Acetonitrile, formic acid, and acetic acid were purchased from Nacalai Tesque Co Ltd. (Kyoto, Japan). Ammonium acetate and ammonia water were purchased from Wako Pure Chemical Industries Co Ltd. (Osaka, Japan). $\beta$-Glucuronidase (E. coli K12 origin; 200 units $/ \mathrm{ml}$ ) was obtained from Roche Diagnostics (Mannheim, Germany). Creatinine test Wako for Jaffe reaction was purchased from Wako Pure Chemical Industries Co Ltd. (Osaka, Japan).

\section{Materials}

All of the glassware used in this study was washed by sonication, rinsed with ultrapure water, dried, and heated at $400^{\circ} \mathrm{C}$. Volumetric glassware was washed similarly except for the $400^{\circ} \mathrm{C}$ heating, which was replaced by methanol rinse.

\section{Analytical procedure}

One milliliter of urine was taken to a glass centrifuge tube to which was then added $300 \mu \mathrm{l}$ mixed internal standard solution $(100 \mathrm{ng} / \mathrm{ml}), 250 \mu \mathrm{l} 1 \mathrm{~mol} / \mathrm{l}$ ammonium acetate buffer ( $\mathrm{pH} 6.5$ ), and $\beta$-glucuronidase ( $5 \mu \mathrm{l})$. Subsequently, urine sample was vortex-mixed, and incubated at $37^{\circ} \mathrm{C}$ for $60 \mathrm{~min}$ for the hydrolysis of glucuronide. After incubation, $3 \mathrm{ml}$ ammonium water ( $\mathrm{pH}$ 8.0) was added to urine samples, and then loaded onto solid-phase extraction cartridge (OASIS-MAX $150 \mathrm{mg} / 6 \mathrm{cc}$, Nihon Waters Co Ltd. Tokyo, Japan), which had been conditioned with acetonitrile $(10 \mathrm{ml})$ and ultrapure water $(5 \mathrm{ml})$. After washing the cartridge with ultrapure water $(5 \mathrm{ml})$ and acetonitrile $(5 \mathrm{ml})$, target monoesters were eluted with $5 \mathrm{ml}$ acetonitrile containing $1 \%$ formic acid to a new glass centrifuge tube. The eluent was evaporated to dryness with a gentle stream of nitrogen gas; the residue was redissolved in $200 \mu \mathrm{l}$ ultrapure water and transferred to an autosampler vial. The nine phthalate monoesters were determined by HPLC-MS/MS. The HPLC was from Agilent technologies (Agilent 1100, CA, USA) and the MS/MS was from Micromass (Micromass Quattro Ultima, Manchester, UK).

Creatinine concentrations in urine samples were determined by Jaffe reaction.
Daily intake estimates

We used Eq. 1 for the estimation of daily intake of seven phthalate diesters from urinary monoester concentrations, as employed by David [22] and Koch et al. [14].

$$
\begin{aligned}
& \text { Daily intake }(\mu \mathrm{g} / \mathrm{kg} / \text { day }) \\
& =\frac{\mathrm{ME}(\mu \mathrm{g} / \mathrm{g}) \times \mathrm{CE}(\mathrm{mg} / \mathrm{kg} / \text { day })}{F_{\mathrm{UE}} \times 1,000(\mathrm{mg} / \mathrm{g})} \times \frac{\mathrm{MW}_{\mathrm{d}}}{\mathrm{MW}_{\mathrm{m}}}
\end{aligned}
$$

ME is creatinine-adjusted monoester concentration, CE is creatinine excretion rate normalized by body weight as $18 \mathrm{mg} / \mathrm{kg}$ per day for women [14]. $\mathrm{F}_{\mathrm{UE}}$ is the molar fraction of the urinary-excreted monoester related to the parent diesters. $\mathrm{F}_{\mathrm{UE}}$ values were as follows: for $\mathrm{MnBP}$, 0.69 was used based on report of Anderson et al. [23]. $\mathrm{F}_{\mathrm{UE}}$ for MMP and MEP were unknown and substituted with that for MnBP. For MEHP, MEHHP, and MEOHP, 0.059, 0.233 , and 0.154 were used, respectively [24]. $\mathrm{F}_{\mathrm{UE}}$ value of 0.0215 was used for MINP based on report of Koch et al. [25]. For MnOP, 0.043 was used from the animal data of Albro and Moore [26]. $\mathrm{MW}_{\mathrm{d}}$ and $\mathrm{MW}_{\mathrm{m}}$ are the molecular weight of phthalate diesters and phthalate monoesters, respectively.

\section{Statistical analyses}

Phthalate metabolite concentrations were corrected for urine volume by urinary creatinine concentration $(\mu \mathrm{g} / \mathrm{g}$ cre). In the following statistical analyses, we used logtransformed phthalate metabolite concentrations adjusted creatinine concentrations because the seven phthalate monoester concentrations in urine samples from the pregnant women were log-normally distributed. To calculate inter- and intraindividual variance in urinary metabolite concentrations through 5-12 weeks, we employed mixed models by using SAS proc mixed version 9.1 (SAS Institute inc., Cary, NC). Intraclass correlation coefficient (ICC) was defined as the proportion of interindividual variance to total variance.

\section{Results}

Analysis of urinary phthalate metabolites

Quantitative recoveries of added metabolites were obtained: $71 \%$ (MINP) to $117 \%$ (MMP) for $3 \mathrm{ng} / \mathrm{ml}$ added level and 96\% (MEP, MINP, MnOP) to $112 \%$ (MMP) for $30 \mathrm{ng} / \mathrm{ml}$ added level. Those values were means of four replicates. The reproducibility of analysis expressed as relative standard deviation ranged from $3.4 \%$ for MEOHP 
to $27.8 \%$ for MEHP. These results indicated that our monoesters analysis was accurate in terms of adequate precision and recoveries. No phthalate metabolites were detected in procedural or travel blanks.

Intra- and interindividual variation

in urinary metabolite concentrations

Four spot urine samples were taken from each of 12 subjects within 5-12 weeks in late pregnancy. Creatinineadjusted phthalate ester metabolite concentrations in the 48 urine samples were analyzed for inter- and intraindividual variance. Since MINP and MnOP were hardly detected in the urines, these metabolites were not included in the statistical analyses. Table 1 shows the results of inter- and intraindividual variance and ICCs in log-transformed

Table 1 Inter- and intraindividual variance and intraclass correlation coefficient (ICC) of each urinary phthalate metabolite concentrations

\begin{tabular}{llll}
\hline Urinary metabolites & Interindividual & Intraindividual & ICC $^{\mathrm{a}}$ \\
\hline MMP & 0.07215 & 0.05477 & 0.569 \\
MEP & 0.06827 & 0.07789 & 0.467 \\
MnBP & 0.06876 & 0.03022 & 0.695 \\
MBzP & 0.06605 & 0.1739 & 0.275 \\
MEHP & 0.0462 & 0.04371 & 0.514 \\
MEHHP & 0.04404 & 0.05944 & 0.426 \\
MEOHP & 0.04009 & 0.05736 & 0.411 \\
\hline
\end{tabular}

$n=48$, four spot urine samples from 12 pregnant women, within 16 weeks intervals during 5-12 sampling period

Log-transformed urinary metabolite concentrations were used in this statistical analysis because the seven phthalate monoester concentrations in 48 single spot urine samples from 12 pregnant women were log-normally distributed

${ }^{\text {a }}$ ICC $=$ Interindividual variance/(interindividual variance + intraindividual variance) creatinine-adjusted urinary metabolite concentrations. The ICCs for seven urinary metabolites were MMP (0.57), MEP (0.47), MnBP (0.69), MBzP (0.28), MEHP (0.51), MEHHP (0.43), and MEOHP (0.41).

Phthalate metabolites levels in Japanese pregnant women

Table 2 shows limit of detection, percentage of subjects with detectable concentration in urine, and median concentrations of urinary metabolites with min-max ranges for the 50 Japanese pregnant women. Limit of detections were $0.008-0.07 \mathrm{ng} / \mathrm{ml}$ as urinary level for the nine phthalate ester metabolites. All of the nine creatinine-unadjusted and creatinine-adjusted urinary metabolite concentrations showed log-normal distribution and, therefore, geometric mean, geometric standard deviation, and median concentration were shown in this table. MMP, MEP, MnBP, MBzP, MEOHP, and MEHHP were detected in $100 \%$ of the subjects. Urinary $\mathrm{MnBP}$ concentration (median $66.6 \mu \mathrm{g} / \mathrm{g}$ cre) was highest among the nine monoesters analyzed while those of MINP and MnOP were low.

Comparison of the estimated daily intake level of phthalate esters based on urinary metabolite levels with other estimates

Table 3 shows estimated daily intake of seven phthalate esters based on urinary metabolite concentrations of the 50 subjects of the present study. The mean estimated intakes of the seven phthalate esters ranged 0.1 to $2 \mathrm{ng} / \mathrm{kg}$ per day. None of the estimated intake levels of the present subjects exceeded the tolerable daily intake (TDI) for DEHP, DnBP, and BBzP (Table 3). The TDIs were based on the reproductive and developmental toxicity of phthalate esters

Table 2 Urinary phthalate metabolite concentrations in 50 pregnant women

\begin{tabular}{|c|c|c|c|c|c|c|c|c|c|c|}
\hline \multirow{3}{*}{$\begin{array}{l}\text { Phthalate } \\
\text { monoesters }\end{array}$} & \multirow{3}{*}{$\begin{array}{l}\text { Limit of } \\
\text { detection }(\mathrm{ng} / \mathrm{ml})\end{array}$} & \multirow{3}{*}{$\begin{array}{l}\text { Detection } \\
\text { rate }(\%)\end{array}$} & \multicolumn{8}{|c|}{ Monoester concentrations } \\
\hline & & & \multicolumn{4}{|c|}{ Unadjusted (ng/ml) } & \multicolumn{4}{|c|}{ Adjusted ( $\mu \mathrm{g} / \mathrm{g}$ cre) } \\
\hline & & & Min & Median & Max & GM (SD) & Min & Median & Max & GM (SD) \\
\hline MEHP & 0.022 & 98 & $<\mathrm{LOD}$ & 3.96 & 70.3 & $3.63(3.89)$ & $<\mathrm{LOD}$ & 5.15 & 67.8 & $4.63(3.32)$ \\
\hline MEHHP & 0.008 & 100 & 1.23 & 10.6 & 89.7 & $10.2(2.57)$ & 3.90 & 10.7 & 164 & 12.9 (1.98) \\
\hline MEOHP & 0.015 & 100 & 0.91 & 11.0 & 132 & $11.0(2.75)$ & 3.46 & 11.9 & 174 & $13.7(2.06)$ \\
\hline MMP & 0.024 & 100 & 0.57 & 6.61 & 464 & $8.13(3.72)$ & 2.16 & 8.15 & 714 & $10.1(3.18)$ \\
\hline MEP & 0.02 & 100 & 0.33 & 7.83 & 1,067 & $8.71(4.07)$ & 1.25 & 9.4 & 1430 & $10.9(3.59)$ \\
\hline $\mathrm{MnBP}$ & 0.066 & 100 & 2.92 & 57.9 & 504 & $52.5(2.75)$ & 11.1 & 66.6 & 211 & $66.9(1.79)$ \\
\hline $\mathrm{MBzP}$ & 0.03 & 100 & 0.26 & 3.74 & 74.5 & $4.57(3.31)$ & 0.69 & 4.37 & 106 & $5.74(2.65)$ \\
\hline MINP & 0.035 & 14 & $<\mathrm{LOD}$ & $<\mathrm{LOD}$ & 2.53 & $<\mathrm{LOD}$ & $<\mathrm{LOD}$ & $<\mathrm{LOD}$ & 3.65 & $<\mathrm{LOD}$ \\
\hline $\mathrm{MnOP}$ & 0.017 & 28 & $<$ LOD & $<\mathrm{LOD}$ & 0.22 & $<\mathrm{LOD}$ & $<\mathrm{LOD}$ & $<\mathrm{LOD}$ & 0.23 & $<\mathrm{LOD}$ \\
\hline
\end{tabular}

All urinary metabolite concentrations were log-normally distributed in 50 pregnant women 
Table 3 Daily phthalate intake levels in 50 pregnant women and comparison with TDI of EFSA and the daily intake levels in pregnant women of Swan et al. [8]

\begin{tabular}{|c|c|c|c|c|c|c|c|}
\hline \multirow{2}{*}{$\begin{array}{l}\text { Phthalate } \\
\text { diesters }\end{array}$} & \multirow{2}{*}{$\begin{array}{l}\text { Urinary } \\
\text { metabolite }\end{array}$} & \multicolumn{4}{|c|}{ Daily intake ( $\mu \mathrm{g} / \mathrm{kg}$ per day) } & \multirow{2}{*}{$\begin{array}{l}\text { TDI }(\mu \mathrm{g} / \mathrm{kg} \\
\text { per day) }\end{array}$} & \multirow{2}{*}{$\begin{array}{l}\text { Swan et al. }[8] \\
\text { Median ( } \mu \mathrm{g} / \mathrm{kg} \text { per day) }\end{array}$} \\
\hline & & Min & Median & Max & GM & & \\
\hline DEHP & MEHP & 0.005 & 2.20 & 29.0 & 1.99 & 50 & 2.37 \\
\hline DEHP & MEOHP & 0.400 & 1.10 & 16.8 & 1.33 & 50 & 2.00 \\
\hline DEHP & MEHHP & 0.554 & 1.90 & 27.9 & 2.20 & 50 & 1.33 \\
\hline $\mathrm{DEHP}^{\mathrm{a}}$ & 3 metabolites & 0.62 & 1.73 & 24.6 & 1.94 & 50 & 1.70 \\
\hline DMP & MMP & 0.061 & 0.229 & 20.1 & 0.285 & - & - \\
\hline DEP & MEP & 0.037 & 0.280 & 42.6 & 0.324 & - & $5.32^{\mathrm{b}}$ \\
\hline DnBP & $\mathrm{MnBP}$ & 0.362 & 2.18 & 6.91 & 2.19 & 10 & $0.67^{\mathrm{b}}$ \\
\hline $\mathrm{BBzP}$ & $\mathrm{MBzP}$ & 0.021 & 0.132 & 3.20 & 0.173 & 500 & $0.35^{\mathrm{b}}$ \\
\hline DINP & MINP & 0.018 & 0.056 & 4.38 & 0.071 & - & - \\
\hline DnOP & $\mathrm{MnOP}$ & 0.007 & 0.026 & 0.135 & 0.011 & - & - \\
\hline
\end{tabular}

$D M P$ dimethyl phthalate, $D E P$ diethyl phthalate, $D n B P$ di- $n$-butyl phthalate, $B B z P$ butylbenzyl phthalate, $D E H P$ di(2-ethylhexyl)phthalate, $D I N P$ di-isononyl phthalate, DnOP di- $n$-octyl phthalate

a The value was based on mean values of DEHP intake from the three DEHP metabolite concentrations for each subject

b These diesters were statistically significant with reduced AGI in the Swan et al. [8] study and calculated by Marsee et al. [41]

References: TDI for DBP [28], DEHP [29], and BBzP [30]

[28-30]. Maximum DEHP and DnBP intake levels of the present subjects accounted for $50 \%$ and $70 \%$ of the TDIs, respectively.

\section{Discussions}

Intra- and interindividual variations in urinary metabolite concentrations

Intra- and interindividual variations in urinary metabolite concentrations were compared based on calculation of ICCs. Except for MBzP, the ICCs were greater than 0.4, which indicated that urine metabolite data were reproducible over the sampling period (5-12 weeks) for most of the phthalates. According to Rosner [34], ICC of $0.4-0.75$ is considered "fair to good" reproducibility. Based on the statistical analysis and higher ICCs, we concluded that the urinary metabolite levels in single spot urine could reflect longer-term (approximately 2-month) exposure level of the corresponding diester of the subject.

To date, a couple of studies have been carried out to evaluate the utility of spot urine for the exposure assessment of phthalate esters by analyzing inter- and intraindividual variance of urinary excretion of metabolites. Our results of ICCs, 0.28 to 0.69 , in urinary metabolite concentrations over 5-12 weeks were at the same levels or relatively higher than those reported. Hauser et al. [19] reported ICCs for MMP (0.27), MEP (0.43), MnBP (0.71), MBzP (0.55), and MEHP (0.54) in 3 months.
Teitelbaum et al. [20] reported MEP (0.26), MnBP (0.35), MBzP (0.62), MEHP (0.29), MEHHP (0.24), and MEOHP (0.23) in 6 months. Hoppin et al. [18], Hauser et al. [19], and Teitelbaum et al. [20] concluded that spot urine was usable for longer-term (up to a couple of months) exposure assessment. On the contrary, the conclusion of Fromme et al. [21] was not similar to ours. They measured concentrations of ten urinary metabolites in 50 subjects during eight consecutive days and analyzed for within-subject variance to find substantial day-to-day variation in urinary monoester levels of the subjects. ICCs were moderate and they concluded that spot urine was not suitable for exposure assessment.

Differences in exposure patterns, including exposure pathway, exposure sources, and exposure timing, might result in such inconsistency among studies on reproducibility of phthalate metabolite concentration in spot urine samples.

Theoretically, utility of spot urine would be limited for long-term exposure assessment of a chemical with short biological half-life, such as phthalate esters, if exposure level varied randomly either interindividually or intraindividually. In spite of this limitation, higher ICCs found in this study and other previous ones suggested that phthalate ester exposure level of an individual was primarily determined by a relatively steady habit (e.g., food and personal care products) of the individual. Until the utility of single spot urine for exposure assessment of phthalate esters is established, however, multiple urine samples may ideally be collected from subjects in epidemiologic studies, as 
Hauser et al. [19] pointed out. This obviously requires cost and effort of both subjects and examiners.

Urinary phthalate metabolite levels

in 50 pregnant women

MEHP, MINP, and MnOP were detected in 98\%, 14\%, and $28 \%$ of subjects, respectively. Frequent detection of urinary metabolites shows that subjects were exposed to various phthalate esters. This may allow us to assume that the general Japanese population is also exposed to them on a daily basis. In the case of MINP, several studies have shown that MINP is not main metabolite of DINP, and is further metabolized to oxidative forms [25, 27]. This may be related to the lower MINP concentrations in this study.

For MMP, MEP, MnBP, MBzP, and MEHP, we compared median phthalate monoester concentrations in the present Japanese subjects with those reported in the previous studies, although the sample size and characteristics of the subject population was not comparable. There were greater differences between our results and literature values for MnBP and MEP (Fig. 1) than those for other phthalate metabolites. Urinary MEP concentration in this study $(9.4 \mu \mathrm{g} / \mathrm{g}$ cre $)$ was lower than those in other countries/ regions by one order of magnitude (Fig. 1). This may be related to the much lower production of DEP in Japan (700 tons in 2002 [35]) than in the USA (11,700 tons in 1988 [2]). On the contrary, urinary MnBP concentration was higher in the present study and in European countries $[14,36]$ than in the USA, except for Hoppin et al.'s [18] study. However, production of DBP was 4,135 tons in Japan [35] and 7,752 tons in USA [3], thus it is unlikely that the difference in MnBP concentration between countries was related to production amount of DBP. Rather the

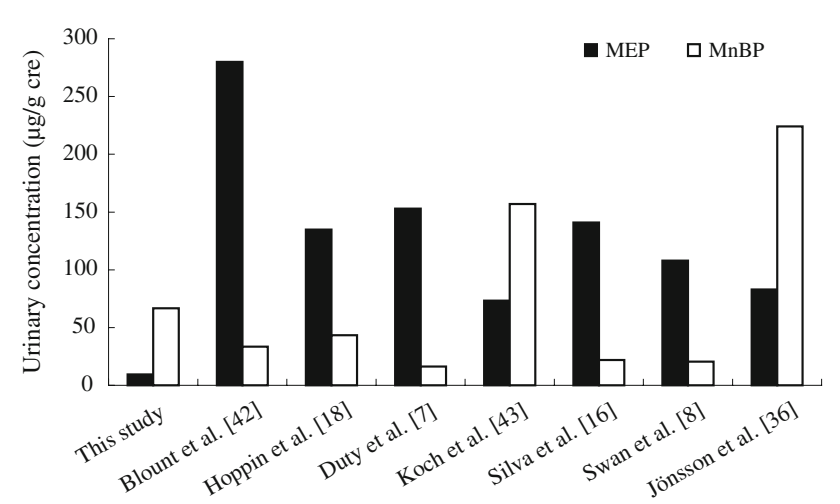

Fig. 1 Comparison of urinary monoester concentrations among recent phthalate exposure assessments with this study. These urinary concentrations were from median values in each study. We compared MEP, MnBP, MMP, MBzP, and MEHP. Comparison results for MEP and $\mathrm{MnBP}$ are shown because of their variations in urinary monoester concentration among regions difference in urinary MnBP concentration between the two countries may reflect the difference in the usage of products containing DBP in the proximate environment of general population.

In contrast, MEHP level in this study was similar to in other studies. Fujimaki et al. [17] measured MEHP, MEOHP, and MEHHP concentrations in spot urine samples from pregnant women in Tokyo and obtained median values of $9.83,10.4$, and $10.9 \mu \mathrm{g} / \mathrm{g}$ cre, respectively, which were of similar order to the present results. The level of MBzP was slightly higher in this study than in others (data not shown). Thus exposure levels of some but not all phthalate esters are different among countries or regions, probably due to differences in use and application of phthalate esters among countries/regions.

Daily intake levels of phthalate diesters and exposure sources

Figure 2 shows comparison of daily exposure level of selected phthalates estimated in this study and those by the Ministry of the Environment (MOE), Japan. The MOE estimate was the sum of the diester intakes from food, indoor air, and drinking water, which were based on the reported mean concentrations in each media in Japan [31-33]. Estimated mean daily intake of DMP, DEP, and DnBP in this study was from two to four times greater than the MOE values, suggesting the presence of exposure sources of these phthalate esters other than food, air, and drinking water for the present subjects (Fig. 2). The difference in the estimation based on urine analysis and environmental monitoring has also been pointed out recently by Itoh et al. [37]. One possible exposure sources that the MOE failed to include was personal care products

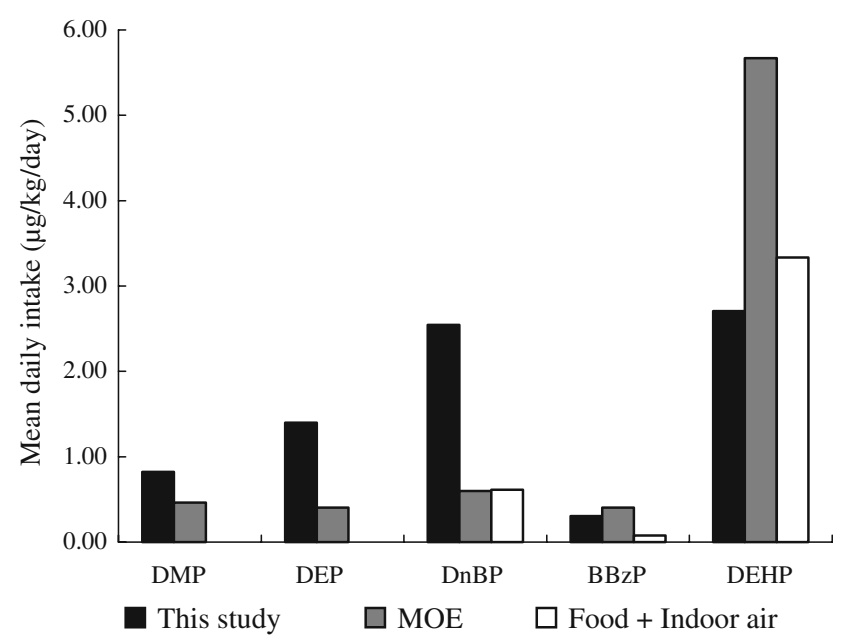

Fig. 2 Mean daily phthalate-intake level in this study, MOE, and estimated intake from food [39] and indoor air [40] for DnBP, BBzP, and DEHP 
such as cosmetics. DEP, DMP, and DBP are the phthalate esters used in personal care products as well as in plastics and others. Considering this use, people are likely to be exposed to DMP, DEP, and DBP, especially via dermal absorption through the usage of cosmetics. Duty et al. [38] reported significant association between urinary MEP concentration and frequency of usage of personal care products. The MOE estimation did not include dermal exposure, which might have resulted in underestimation of exposure. The contribution from personal care products has to be quantitatively estimated for Japanese population to fully assess exposure level of phthalate esters.

On the contrary, our estimated intake of DEHP was lower than the MOE estimation (Fig. 2). In 2000, the Ministry of Health and Welfare of Japan restrained DEHP use in polyvinyl chloride (PVC) gloves because it was suspected that handling of foods with PVC gloves contaminated foods with DEHP. After this regulation, DEHP concentration in food decreased and average daily intake of DEHP decreased from $519 \mu \mathrm{g} /$ day in 1999 to $160 \mu \mathrm{g} /$ day in 2001 [39]. Since the MOE used DEHP concentration in food reported in 1998 for intake estimation, this might have resulted in overestimation of daily DEHP intake level. When we used newer exposure information of DEHP from food [39] and indoor air [40], calculated DEHP intake was $3.33 \mu \mathrm{g} / \mathrm{kg}$ per day, which was consistent with the present estimations from urinary metabolite concentration (1.1$2.2 \mu \mathrm{g} / \mathrm{kg}$ per day). This consistency may support our notion that DEHP exposure from food had recently decreased substantially in Japan.

Exposure levels to phthalate esters

in 50 pregnant women

As shown in Table 3, our estimates of daily intakes of phthalate diesters were lower than TDIs set by the EFSA based on reproductive and developmental effects to offspring in animal experiment. Swan et al. [8] observed relationships between prenatal phthalate exposure and anogenital distance (AGD) of male infants and indicated that humans might be more sensitive to prenatal exposure to phthalate esters than were rodents. The phthalate exposure levels in this study were similar to those in US pregnant women who had male infants with reduced AGD by prenatal phthalate exposure in the Swan et al. study [8] (Table 3). Therefore the present phthalate ester exposure levels might not completely be effect-free, although the intake levels of phthalate esters in this study were lower than TDI values set by the EFSA.

The present study revealed the fact that metabolites of various phthalate esters are detected in urine samples collected from Japanese pregnant women, showing that they were exposed to phthalate esters on a daily basis. It is not clear whether this exposure level of Japanese pregnant women is safe enough for their offspring, though the level was less than TDIs. Further studies are needed to reveal the toxicity of phthalate esters to fetus and other sensitive subpopulations. Exposure monitoring of sensitive subpopulation to phthalates has to be continued as well.

Acknowledgments The authors greatly appreciate K. Omura and H. Nitta, National Institite for Environmental Studies, for their advice on statistical analysis. The authors sincerely appreciate the subjects of this study for supplying urine samples and also the technical staff of the Central Hospital of Self-Defense Force for sampling.

\section{References}

1. ATSDR (Agency for Toxic Substances and Disease Registry) Toxicological profile for di(2-ehylhexyl) phthalate. Available at: http://www.atsdr.cdc.gov/toxprofiles/tp9.html, 2002.

2. ATSDR (Agency for Toxic Substances and Disease Registry) Toxicological profile for diethyl phthalate. Available at: http://www.atsdr.cdc.gov/toxprofiles/tp73.html, 1995.

3. ATSDR (Agency for Toxic Substances and Disease Registry) Toxicological profile for di-n-butyl phthalate. Available at: http://www.atsdr.cdc.gov/toxprofiles/tp135.html, 2001.

4. Matsumoto M, Koizumi M, Ema M. Potential adverse effects of phthalic acid esters on human health: a review of recent studies on reproduction. Regul Toxicol Pharmacol. 2008;50:37-49.

5. Gray LE Jr, Ostby J, Furr J, Price M, Veeramachaneni DN, Parks L. Perinatal exposure to the phthalates DEHP BBP, and DINP, but not DEP, DMP, or DOTP, alters sexual differentiation of the male rat. Toxicol Sci. 2000;58:350-65.

6. Foster PMD. Effects of phthalate esters on the developing reproductive tract of male rats. Hum Reprod Update. 2001;7:231-5.

7. Duty SM, Silva MJ, Barr DB, Brock JW, Ryan L, Chen Z, et al. Phthalate exposure and human semen parameters. Epidemiology. 2003; 14:269-77.

8. Swan SH, Main KM, Liu F, Stewart SL, Kruse RL, Calafat AM, et al. Decrease in anogenital distance among male infants with prenatal exposure. Environ Health Perspect. 2005;113:1056-61.

9. Main KM, Mortensen GK, Kaleva MM, Boisen KA, Damgaard IN, Chellakooty M, et al. Human breast milk contamination with phthalates and alterations of endogenous reproductive hormones in infants three months of age. Environ Health Perspect. 2006;114:270-6.

10. Schmid P, Schlatter C. Excretion and metabolism of di(2-ethylhexyl)-phthalate in man. Xenobiotica. 1985;15:251-6.

11. Silva MJ, Barr DB, Reidy JA, Kato K, Malek NA, Hodge CC, et al. Glucuronidation patterns of common urinary and serum monoester phthalate metabolites. Arch Toxicol. 2003;77:561-7.

12. Blount BC, Milgram KE, Silva MJ, Malek NA, Reidy JA, Needham LL, et al. Quantitative detection of eight phthalate metabolites in human urine using HPLC-MS/MS. Anal Chem. 2000;72:4127-34

13. Silva MJ, Malek NA, Hodge CC, Reidy JA, Kato K, Barr DB, et al. Improved quantitative detection of 11 urinary phthalate metabolites in humans using liquid chromatography-atmospheric pressure chemical ionization tandem mass spectrometry. J Chromatogr B. 2003;789:393-404.

14. Koch HM, Drexler H, Angerer J. An estimation of the daily intake of di(2-ethylhexyl)phthalate (DEHP) and other phthalates in the general population. Int $\mathrm{J}$ Hyg Environ Health. $2003 ; 206: 77-83$. 
15. Duty SM, Singh NP, Silva MJ, Barr DB, Brock JW, Ryan L, et al. The Relationship between environmental exposures to phthalates and DNA damage in human sperm using the neutral comet assay. Environ Health Perspect. 2003;111:1164-9.

16. Silva MJ, Barr DB, Reidy JA, Malek NA, Hodge CC, Caudill SP, et al. Urinary levels of seven phthalate metabolites in the U.S. population from the National Health and Nutrition Examination Survey (NHANES) 1999-2000. Environ Health Perspect. 2004;112:331-8.

17. Fujimaki K, Yoshinaga J, Watanabe C, Serizawa S, Shiraishi H, Mizumoto Y. Estimation of intake level of di(2-ethylhexyl) phthtalate (DEHP) of Japanese pregnant woman based on measurement of concentrations of three urinary metabolites. Nippoon Eiseigaku Zasshi. 2006;61:340-7. (in Japanese).

18. Hoppin JA, Brock JW, Davis BJ, Baird DD. Reproducibility of urinary phthalate metabolites in first morning urine samples. Environ Health Perspect. 2002;110:515-8.

19. Hauser R, Meeker JD, Park S, Silva MJ, Calafat AM. Temporal variability of urinary phthalate metabolite levels in men of reproductive age. Environ Health Perspect. 2004;112:1734-40.

20. Teitelbaum SL, Britton JA, Calafat AM, Ye X, Silva MJ, Reidy JA, et al. Temporal variability in urinary concentrations of phthalate metabolites, phytoestrogens and phenols among minority children in the United States. Environ Res. 2008;106:257-69.

21. Fromme H, Bolte G, Koch HM, Anger J, Boehmer S, Drexler H, et al. Occurrence and daily variation of phthalate metabolites in the urine of an adult population. Int J Hyg Environ Health. 2007;210:21-33.

22. David RM. Exposure to phthalates. Environ Health Perspect. 2000;108:A440.

23. Anderson WA, Castle L, Scotter MJ, Massey RC, Springall C. A biomarker approach to measuring human dietary exposure to certain phthalate diesters. Food Addit Contam. 2001;18:1068-74.

24. Koch HM, Bolt HM, Preuss R, Angerer J. New metabolites of di(2-ethylhexyl)phthalate (DEHP) in human urine and serum after single oral doses of deuterium-labelled DEHP. Arch Toxicol. 2005;79:367-76.

25. Koch HM, Angerer J. Di-iso-nonylphthalate (DINP) metabolites in human urine after a single oral dose of deuterium-labelled DINP. Int J Hyg Environ Health. 2007;210:9-19.

26. Albro PW, Moore B. Identification of the metabolites of simple phthalate diesters in rat urine. J Chromatogr. 1974;94:209-18.

27. Silva MJ, Reidy JA, Preau JL Jr, Needham LL, Calafat AM. Oxidative metabolites of diisononyl phthalate as biomarkers for human exposure assessment. Environ Health Perspect. 2006; 114:1158-61.

28. European Food Safety Authority (EFSA). Opinion of the Scientific Panel on Food Additives, Flavourings, Processing Aids and Materials in Contact with Food (AFC) on a request from the Commission related to Di-Butylphthalate (DBP) for use in food contact materials. EFSA J. 2005;242:1-17.
29. EF SA. Opinion of the Scientific Panel on Food Additives, Flavourings, Processing Aids and Materials in Contact with Food (AFC) on a request from the Commission related to Bis(2-ethylhexyl)phthalate (DEHP) for use in food contact materials. EFSA J. 2005;243:1-20.

30. SA EF. Opinion of the Scientific Panel on Food Additives, Flavourings, Processing Aids and Materials in Contact with Food (AFC) on a request from the Commission related to Butylbenzylphthalate $(\mathrm{BBzP})$ for use in food contact materials. EFSA J. 2005;241:1-14.

31. Japan Ministry of the Environment. Environmental risk assessment of chemicals vol. 1 Available at: http://www.env.go.jp/chemi/ report/h14-05/index.html, 2002 (in Japanese).

32. Japan Ministry of the Environment. Environmental risk assessment of chemicals vol. 3 Available at: http://www.env.go.jp/ chemi/report/h16-01/index.html, 2004 (in Japanese).

33. Japan Ministry of the Environment. Environmental risk assessment of chemicals vol. 4 Available at: http://www.env.go.jp/ chemi/report/h17-21/index.html, 2005 (in Japanese).

34. Rosner B. Fundamentals of biostatistics. 6th ed. CA: Duxbury; 2006.

35. The Chemical Daily. 14504 chemical products. Tokyo: The Chemical Daily Co, Ltd.; 2004. (in Japanese).

36. Jönsson BA, Richthoff J, Rylander L, Giwercman A, Hagmar L. Urinary phthalate metabolites and biomarkers of reproductive function in young men. Epidemiology. 2005;16:487-93.

37. Itoh $\mathrm{H}$, Toshida $\mathrm{K}$, Masunaga S. Quantitative identification of unknown exposure pathways of phthalates based on measuring their metabolites in human urine. Environ Sci Technol. 2007; 41:4542-7.

38. Duty SM, Ackerman RM, Calafat AM, Hauser R. Personal care product use predicts urinary concentrations of some phthalate monoesters. Environ Health Perspect. 2005;113:1530-5.

39. Tsumura Y, Ishimitsu S, Saito I, Sakai H, Tsuchida Y, Tonogai Y. Estimated daily intake of plasticizers in 1-week duplicate diet samples following regulation of DEHP-containing PVC gloves in Japan. Food Addit Contam. 2003;20:317-24.

40. Otake T, Yoshinaga J, Yanagisawa Y. Exposure to phthalate esters from indoor environment. J Expo Anal Environ Epidemiol. 2004;14:524-8.

41. Marsee K, Woodruff T, Axelrad D, Calafat A, Swan H. Estimated daily phthalate exposures in a population of mothers of male infants exhibiting reduced anogenital distance. Environ Health Perspect. 2006;114:805-9.

42. Blount BC, Silva MJ, Caudill SP, Needham LL, Pirkle JL, Sampson EJ, et al. Levels of seven urinary phthalate metabolites in a human reference population. Environ Health Perspect. 2000;108:979-82.

43. Koch HM, Rossbach B, Drexler H, Angerer J. Internal exposure of the general population to DEHP and other phthalates-determination of secondary and primary phthalate monoester metabolites in urine. Environ Res. 2003;93:177-85. 